\title{
Jean Van Heijenoort: Una vida azarosa, digna de muchas novelas.
}

\author{
Vernor Arguedas \\ vernor.arguedas@ucr.ac.cr \\ Escuela de Matemática \\ Universidad de Costa Rica \\ Recibido: Febrero 15, $2016 \quad$ Aceptado: Febrero 29, 2016
}

Resumen. Jean Van Heijenoort y su vida como un joven brillante, guardaespaldas de Trotsky, después matemático y lógico destacado. Uno de los responsables de los archivos de la Universidad de Harvard sobre Trotsky.

Palabras clave: Jean Van Heijenoort, Trotsky, Frida Kahlo, conjuntos convexos, Frege, Gödel, lógica..

Abstract. Jean Van Heijenoort and his life, a brilliant young man, bodyguard of Trotsky. Later on he turned into a prominent mathematician and logician, and one of those responsible for the archives of the University of Harvard on Trotsky.

KeyWords: Jean Van Heijenoort , Trotsky, Frida Kahlo, convex sets, Frege, Gödel ,logic.

\subsection{Introducción}

Iniciamos esta nota con una referencia de Laurent Schwartz, el creador de la teoría de las distribuciones y ganador de la medalla Fields en 1950, sobre Jean Van Heijenoort en su libro: "Un Matemático Enfrentado a su Siglo" (traducción libre del título) [1]. Una parte de la cita en cuestión dice (traducción libre):

"Uno de nuestros camaradas, Jean Van Heijenoort acompañó a Trotsky en el exilio. Él era un joven francés de origen modesto quien había perdido a su padre a muy temprana edad. Excelente estudiante iba a ingresar a la ENS (École Normale Superieure) para estudiar matemáticas. Conoció a Ivan Craipeau que lo convenció de hacerse trotskista. Decidió entonces que la revolución mundial era más importante que sus estudios y en 1932 se unió a Trotsky en Prinkipo, luego en Francia y después en México. El permaneció hasta 1939 como su guardaespaldas y secretario. En el partido francés 
hablábamos frecuentemente del camarada Van Heijenoort, un fornido compañero con grandes habilidades que se había convertido en un revolucionario profesional, como el mismo Trotsky."

Juan Forn en escribe una emotiva semblanza de Jean Van Heijenoort. En una parte leemos [2]:

"Jan Van Heijenoort tenía un don para la matemática: podía resolver de un golpe de vista ecuaciones con tres incógnitas. Por esa razón recibió beca completa para el Lycée St-Louis de París, pero no fue por eso que se convirtió en secretario, traductor y guardaespaldas de León Trotsky cuando acababa de cumplir veinte años".

Anita Burdman Feferman (2007-2015) escribió un excelente libro sobre Jan Van Heijenoort; "Politics, Love and Logic" [3] basado en entrevistas con Jan Van Heijenoor y otras fuentes. Usualmente sus amistades lo llamaban "el del nombre impronunciable " y se referían a él simplemente como "Van". En este material se ilustran diversas facetas de su azarosa vida y su trágica muerte. Jan Van Heijenoort nació en Creil, Francia, en 1912 y murió en la ciudad de México en 1986. Su padre era un emigrante holandés quien falleció cuando Van Heijenoort tenía dos años de edad. Las condiciones en las que pereció su padre y los terribles acontecimientos de la primera guerra mundial lo marcaron para siempre. Gracias a su extraordinaria inteligencia pudo recibir una educación formal en el estilo francés por medio de becas. En Paris se afilió a los círculos trotskistas, a sus veinte años era ya un poliglota consumado, incluso había aprendido ruso de manera autodidacta. Cuando recibió el llamado de Trotsty no lo pensó dos veces.
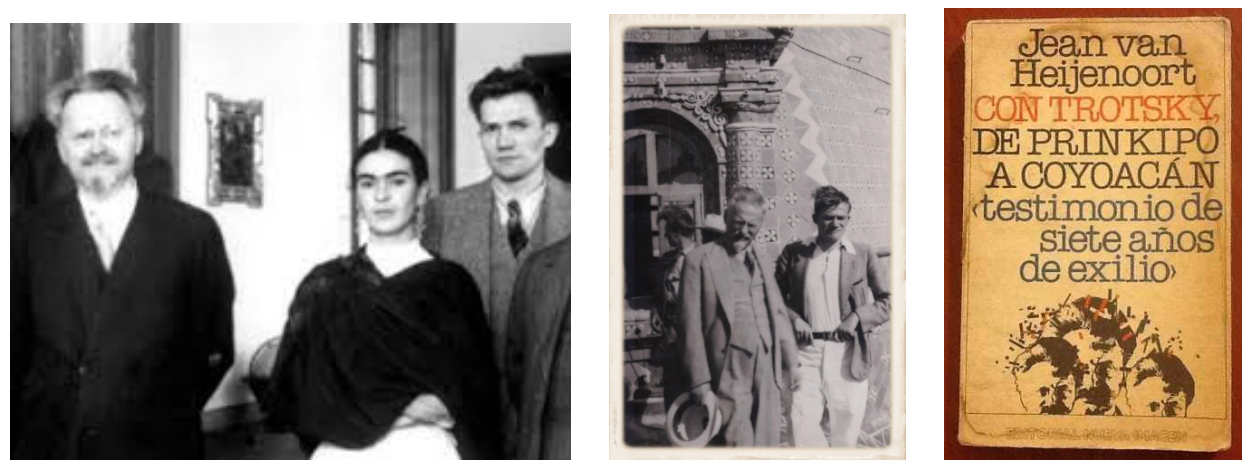

\subsection{Trotsky}

Detalles de su vida se encuentran en la autobiografía titulada: "With Trostky in Exile: From Prinkipo to Coyoacan", publicado en 1978 por Harvard University Press, traducida al castellano por Tununa Mercado,1979. Hay una versión libre preparada para Marxists.org por Rodrigo Cisterna en 2013 que se puede obtener en [5]. En la nota introductoria leemos:

Los recuerdos del secretario, traductor y guardaespaldas de Trotsky entre octubre de 1932 y noviembre de 1939 recrean detalladamente la atmósfera en que vivía y trabajaba en esos años de exilio. El relato simple y preciso de esa cotidianeidad trascendente permite, en no pocos casos, superar errores involuntarios de otros autores, disipar calumnias y despojar al personaje del aura mitológica que, como a todos los grandes hombres, suele creársele. Este libro, lejos de basarse en la frágil memoria, está minuciosamente verificado con un archivo personal del autor que contiene 22 mil documentos 
(entre ellos 4 mil cartas de Trotsky), correspondientes al periodo que se extiende entre 1929 y 1940. El periodo del exilio en México es ampliamente considerado, aportando mucha -y nueva- información, de quien fuera testigo de la relación con Diego Rivera, Frida Kahlo, Bretón y gran cantidad de personalidades mexicanas. Eludiendo la devoción incondicional tanto como la hostilidad sistemática, el relato de Heijenoort -que no pretende ser un examen integral de la personalidad de Trotsky, de sus ideas y de su carácter- contribuye sin embargo a la visión crítica de una etapa histórica que los sucesos posteriores actualizan hoy. Una experiencia vivida no se transmite como un objeto. Su transmisión es una reconstrucción, una reconstrucción para quien escribe y otra, quizás diferente, para quien lee. De eso se trata

Viví junto a León Trotsky, salvo algunas interrupciones, de octubre de 1932 a noviembre de 1939. Era miembro de su organización política y me convertí en su secretario, traductor y guardaespaldas. El pequeño libro que presento no es la historia política de esos años. Tampoco es un retrato de cuerpo entero del hombre. Son recuerdos, mis recuerdos. Intento recrear la atmósfera en la que vivía y trabajaba Trotsky durante esos años de exilio. Me esforzaré por no repetir aquí lo que ya se conoce y lo haré sólo cuando sea necesario para apoyar mi relato. Pido en consecuencia a mi lector cierto conocimiento de los acontecimientos de que hablo. Le pido también que a veces restablezca ciertas proporciones; mi relato, muy a menudo, estará hecho de detalles puesto que soy el único que los conoce y no quiero que desaparezcan conmigo; el lector no deberá perder nunca de vista el contexto en el que todo eso debe insertarse. En el transcurso de mi relato sucederá que a veces tenga que dar un detalle que, a primera vista, podrá parecer de un interés menor. Es que sé, por mi conocimiento del pasado y de los archivos, que esa información quizás permitirá que un investigador pueda reconstituir un hecho, identificar un documento. Lo que se ha escrito sobre León Trotsky después de su muerte, aun cuando sus autores hayan sido personas de buena voluntad, contiene una buena dosis de errores materiales. En un Apéndice trato de corregir algunos de esos errores. Pero a menudo mi propio texto, sin que yo señale explícitamente un error, ha sido escrito como reacción contra algún fragmento de un trabajo que considero erróneo. Sobre aquellos episodios que peor se conocen es donde más me extiendo e intento dar todos los detalles que recuerdo. Ciertos errores que se han vuelto tan frecuentes a menudo sólo son faltas debidas a distracciones, a veces a tonterías. Pero Trotsky es un personaje que parece destinado a provocar actividades mitogénicas, contra las que creo que se debe reaccionar presentando un relato lo más preciso y concreto posible. Por añadidura, las calumnias stalinistas contra Trotsky fueron tan masivas, tan prolongadas, que seguramente quedan aquí y allá algunos restos de mal olor; la mejor manera de disiparlos es contar simple y exactamente cómo vivía. Conozco demasiado bien las flaquezas de la memoria para llegar a imaginar que no hay errores en mi relato. Pero había guardado algunas notas y tuve a mi disposición los archivos, archivos que yo mismo había puesto en orden. Pude verificar entonces bastantes cosas. No he creído oportuno mezclar a estos recuerdos un examen crítico de la personalidad de León Trotsky, de sus ideas y de su carácter. Ésa sería otra tarea. Los archivos contienen, tan sólo para el período de 1929 a 1940, cerca de 22 mil documentos. En otras partes se descubrirán otros. Entre esos documentos hay cerca de cuatro mil cartas de Trotsky, quien fue un gran cultor de la epístola tanto por la cantidad como por el estilo. Todo eso queda por explotar. Hasta ahora los escritos de Trotsky han sido objeto, casi exclusivamente, ya sea de una anatematización completa, o de una veneración devota. Pero lo que esos textos piden es una crítica. Crítica de las ideas y de sus encadenamientos, de los argumentos empleados, de las perspectivas y de sus cambios. Crítica literaria también, con un examen del estilo, un estudio de las metáforas que puedan conducir a apreciaciones sobre la persona del autor. Todo eso queda por hacer, pero no es lo que quise emprender en mi libro. Lo que aporto en este pequeño volumen son, en cierta medida, algunos materiales para ese trabajo. Demasiadas veces me sucedió que al contar tal o cual episodio de mi vida con Trotsky mi interlocutor sacara conclusiones muy diferentes de las que yo pensaba obtener con mi relato, como para no saber que el poder de las palabras tiene sus límites. He tratado de elegir bien las mías, pero sin forjarme muchas ilusiones. Sin duda habrá malentendidos. Una experiencia vivida no se transmite como un objeto. Su transmisión es una reconstrucción, reconstrucción para 
quien escribe y reconstrucción, quizás diferente, para quien lee. Dicho esto, he aquí mi relato

El libro es muy interesante y en la referencia indicada anteriormente se puede leer completo. La nota periodística de Juan Forn [2] comenta:

Con la llegada de Hitler al poder en Alemania a Trotsky se le abrió un nuevo frente y se le cerró la entrada de los únicos derechos de autor más o menos confiables que recibía hasta entonces. Ahora, además de denunciar las maniobras de Stalin, debía precaver al mundo de que Hitler llevaría a Europa a la guerra. También debió irse de Prinkipo, de incógnito: primero a Francia, después a Noruega, después a México. Siempre con la misma rutina: escasez de recursos, trabajo febril, vigilancia insomne, malas noticias constantes. Los voluntarios se iban fundiendo y eran reemplazados. Todos menos Van. Cuando Trotsky se perdió en un bosque en Noruega, Van lo salvó de morir de frío. Cuando a Trotsky se le desbocó el caballo en Cuernavaca, Van lo corrió y lo rescató (aunque era la primera vez que montaba en su vida). Cuando a Trotsky no le daba más la cabeza y era necesario terminar el trabajo igual, sólo confiaba en Van, fuese un artículo de prensa, una carta confidencial o un asunto de polleras (hay quien dice que el romance que tuvo Van con Frida Kahlo fue para sacársela de encima a Trotsky). Cuando la mujer de Van tuvo un cruce de palabras con la señora Trotsky en la cocina, Van la fletó a París (y como ella estaba embarazada, Van recién pudo conocer a su hija años después). Cuando Trotsky y señora recibieron la noticia de la muerte de sus hijos (el suicidio de Zina, primero, cuando estaban en Prinkipo, y el envenenamiento de Liova cuando ya estaban en México), la reacción fue la misma, encerrarse en su dormitorio durante tres días, y Van era el encargado de pasarles té por la puerta entreabierta, el único autorizado a acercarse

\subsection{Estados Unidos}

En noviembre de 1939 la vida de Jean Van Heijenoort da un giro total: por instrucciones de Trotsky viaja a Estados Unidos de Norteamérica.

Trotsky le indicó que había estado tantos años a su sombra que era necesario que viviera un poco por sí mismo, y lo envió a estudiar la situación interna del Socialist Workers Party, el partido trotskista norteamericano. Vivía en pensiones, hacía arreglos de plomería para pagarse el traslado de ciudad en ciudad mientras preparaba concienzudamente su informe. En las calles de Baltimore se enteró por los diarios del asesinato de Trotsky (21 de agosto 1940) y se derrumbó. "Sólo el estudio de las matemáticas me permitió conservar el equilibrio interior", dijo en un libro que escribió cuarenta años después. El libro era sobre Trotsky, aunque Van era para entonces profesor emérito de matemáticas y lógica en Harvard y en Stanford, con oficina propia en ambas costas.

A los treinta y tres años, luego del fin de la guerra, logró entrar en los cursos gratuitos de la universidad pública de Nueva York. Se graduó y después se doctoró, primero en matemática y a continuación en lógica. Fue el único capaz de poner en orden los papeles póstumos de $\mathrm{G}$ del, una tarea considerada titánica y decisiva en el mundo de la lógica. Seguía trabajando veinte horas al día, como en los tiempos de Prinkipo, sólo que ahora dedicaba doce horas a la matemática y apenas ocho a Trotsky. Mientras sus colegas académicos descansaban de las labores diarias, él se dedicaba a rastrear, clasificar, traducir y ordenar todos los papeles de Trotsky diseminados en las accidentadas etapas del exilio. Logró que

GJean Van Heijenoort Una vida azarosa, digna de muchas novelas.. Vernor Arguedas

Derechos Reservados (C) 2016 Revista digital Matemática, Educación e Internet (http://tecdigital.tec.ac.cr/revistamatematica/) 
Harvard comprara esos miles de documentos y abriera un archivo sobre Trotsky. La tesis doctoral de Van Heijenoort es en geometría diferencial sobre superficies convexas. En el artículo publicado originalmente en 1952: "On locally convex manifolds - Van Heijenoort" [6], se pueden apreciar sus grandes dotes de matemático; esta publicación, puesta tardíamente en línea, continúa la investigación de su tesis.

En 1948 Van Heijenoort abandona el trotskismo y escribe lo siguiente (en inglés).

By 1948, the Marxist-Leninist ideas about the proletariat and its political capacity seemed more and more to me to disagree with reality ... I pondered my doubts, and for several years the study of mathematics was all that allowed me to preserve my inner equilibrium. Bolshevik ideology was, for me, in ruins. I had to build another life.

A continuación una traducción libre:

En 1948 las ideas marxistas-leninistas acerca del proletariado y su capacidad política me parecieron más y más alejadas de la realidad. Medité sobre mis dudas y por varios años fue el estudio de las matemáticas lo único que me permitió mantener mi equilibrio interno. La ideología bolchevique estaba para mí en ruinas. Tenía que construir otra vida. (Tomado de [4])

En marzo de 1948 Van hace una crítica muy fuerte al papel del proletariado, en el aniversario 100 de la publicación del Manifiesto Comunista. Lo escribe bajo el seudónimo de Jean Vannier [9]

En [10] se encuentran algunos de los trabajos de Van Heijenoort sobre diversos temas políticos desde un punto de vista marxista; comprende el periodo entre 1940 y 1948. Muchas de estas publicaciones están escritas bajo otro nombre pues acostumbraba utilizar seudónimos con frecuencia.

En esa lista aparece una crítica feroz ( muy bien justificada) contra las concepciones matemáticas de Engels. Se titula: "Friedrich Engels and Mathematics" [7]. En el libro antes citado de Laurent Schwartz, este ilustrísimo científico hace algo parecido con los conocimientos matemáticos de Marx.

\subsection{Matemáticas y Lógica}

Van Heijenoort desarrolló una prolífica vida académica como matemático y después como lógico. Sus trabajos sobre Frege y Gödel son extraordinarios. Una aproximación a su interés en lógica se obtiene de la lectura de artículo: "Lógica como Cálculo y Lógica como Lenguaje". Se puede leer y descargar en formato pdf en [8]. La versión original está escrita en inglés y se titula: "Logic as Calculus and Logic as Languaje", Synthese, Vol.17,No.3 (1963).

$\mathrm{Su}$ aporte a la lógica y a la historia de esa disciplina se refleja en muchos de sus libros especialmente en: "Frege and Gödel: Two fundamental texts in mathematical logic", "From Frege to Gödel : a source book in mathematical logic" y "Gödel, Kurt: Collected works / Kurt Gödel".

GJean Van Heijenoort Una vida azarosa, digna de muchas novelas.. Vernor Arguedas

Derechos Reservados (C) 2016 Revista digital Matemática, Educación e Internet (http://tecdigital.tec.ac.cr/revistamatematica/) 


\subsection{Amores difíciles}

A través de su vida, el apuesto y reservado Van Heijenoort mantuvo relaciones sentimentales apasionadas, difíciles y hasta peligrosas. A su primera esposa, Gabrielle Brausch, la conoció en el grupo trotskista francés. Ella fue la madre de su primer hijo, Jeannot. Su dedicación al trabajo con Trotsky lo alejó de su primera familia.

Mientras residía en México, vivió una furtiva pasión con la atractiva y seductora Frida Kahlo, a quien calificó como "una de las grandes mujeres" de su vida. En ese país, conoció a la trotskista Loretta Guyer, natural de Nueva York, con quien se casó en 1939 y lo hizo padre de una niña: Laure.

Su tercera esposa fue Anne B., una bella estadounidense veinte años más joven que él. Este nuevo matrimonio naufragó por diferencias irreconciliables. En un viaje a México en 1958, Van se encontró con Ana María Zamora, a quien había conocido cuando era pequeña: ella era la hija de Adolfo Zamora, el abogado mexicano de Trotsky. Con Ana María anudó un vínculo sentimental que lo llevaría a casarse por cuarta vez, en 1969 (se divorciarían al cabo de 12 años para volver a unirse en matrimonio tres años después). Esta unión, inicialmente, fue venturosa, pero al cabo de un tiempo se hizo evidente que sus expectativas no coincidían. A la vez, la inestabilidad emocional de Ana María fue un factor que influyó en el deterioro de la relación.

\subsection{Un trágico final}

En 1986, Van trabajaba en un proyecto editorial para la Universidad de Stanford cuando le fue comunicado que su esposa atravesaba por una profunda crisis psicológica. De inmediato, se trasladó a Ciudad de México, creyendo que su presencia la aliviaría.

En la noche del 28 de marzo, mientras Van Heijenoort dormía en el estudio de la casa de su esposa, en la exclusiva colonia de Lomas de Chapultepec, tres disparos de bala impactaron en su cabeza. Ana María Zamora presionó el gatillo una vez más para acabar con su propia vida. En varias ocasiones ella había amenazado con suicidarse y eliminar a Van si éste la dejaba. La amenaza se había cumplido.

Jan Van Heijenoort está enterrado en una tumba del Panteón Francés en el DF. La tumba de Trotsky está cerca, con sus conejeras y su museo.

\section{Bibliografía}

[1] Laurent Schwartz (2001). A Mathematician Grappling with His Century. Birkhäuser Basel. http:// libgen .io/ads . php?md5=d22eb5aa9743de04a0a56a33609cceda

[2] Juan Forn. "El secretario tenaz". http://www.pagina12.com.ar/diario/contratapa/13-2617562014-12-12.html 
[3] Anita Burdman Feferman (1993). Politics, Logic, and Love: The Life of Jean Van Heijenoort. Jones and Bartlett.

[4] Anita Burdman Feferman (2000). From Trotsky to Gödel: The Life of Jean van Heijenoort. A K Peters/CRC Press; 1 edition.

[5] Rodrigo Cisterna (2013. "Con Trotsky en el exilio: De Prinkipo a Coyoacán". https://www. marxists.org/espanol/heijenoort/1978/exilio.htm

[6] John Van Heijenoort (1952). On locally convex manifolds. DOI: 10.1002/cpa. 3160050302

[7] John Van Heijenoort (1948). "Friedrich Engels And Mathematics". https://www.marxists.org/ history/etol/writers/heijen/works/math.htm

[8] John Van Heijenoort (1967). "Logic as calculus and logic as language", Synthese, 17, Issue 1 (1967) pp. 324-330. https://docs.google.com/viewer?a=v\&pid=sites\&srcid= ZGVmYXVsdGRvbWFpbnxkcHRvZmlsb3NvZmlhaXBhfGd4OmE5NGI3NDdlOGVkNTQ3Zg

[9] Jean Vannier (1948). "A Century's Balance Sheet". https://www.marxists.org/history/etol/ writers/heijen/1948/03/balancesheet.html

[10] ETOL Writers. "Jean van Heijenoort". https://www.marxists.org/history/etol/writers/ heijen/index.htm 